\title{
Synthetic Mudscapes: Human Interventions in Deltaic Land Building
}

\author{
Elizabeth Anne Williams, Jeff Carney, Bradley Cantrell, Matt Seibert, Katrina Durbak, Patrick Michaels, \\ Silvia Cox, Ian Miller, Ben Hartman, Gyan Basyal, Emily Powell, Valeria Guida and Clay Tucker \\ The Coastal Sustainability Studio, Louisiana State University, USA
}

\begin{abstract}
In order to defend infrastructure, economy, and settlement in Southeast Louisiana, we must construct new land to mitigate increasing risk. Links between urban environments and economic drivers have constrained the dynamic delta landscape for generations, now threatening to undermine the ecological fitness of the entire region. Static methods of measuring, controlling, and valuing land fail in an environment that is constantly in flux; change and indeterminacy are denied by traditional inhabitation.

Multiple land building practices reintroduce deltaic fluctuation and strategic deposition of fertile material to form the foundations of a multi-layered defence strategy. Manufactured marshlands reduce exposure to storm surge further inland. Virtual monitoring and communication networks inform design decisions and land use becomes determined by its ecological health. Mudscapes at the threshold of land and water place new value on former wastelands. The social, economic, and ecological evolution of the region are defended by an expanded web of growing land.
\end{abstract}

Keywords: Risk, Coastal, Adaptive, Mudscapes

\section{SUBHEAD REQUIRED}

The Coastal Sustainability Studio is a transdisciplinary research and design operation at Louisiana State University. Including architects, landscape architects, urban planners, civil engineers, and coastal scientists, the workshop is a collaborative effort incorporating the College of Art+Design, the College of Engineering, and the School of the Coast \& Environment. The studio addresses issues stemming from coastal erosion and wetland disintegration, incorporating the needs of specific design projects and their relationships with ecological, institutional, industrial, and socioeconomic systems amongst the built environment. The works of CSS aim to reduce economic losses and protect assets, promote a sustainable coastal system by incorporating natural and constructed processes, provide sustainable habitats to support an array of commercial and recreational heritage, and sustain the unique coastal heritage of the state of Louisiana.

Along with increased storm frequencies and intensities, coastal environments around the world are facing growing challenges of subsidence, land loss, saltwater intrusion, and sea level rise. These zones at the thresholds of land and water are often densely inhabited, both by spectrums of urban settings and the industries and infrastructures that sustain them. Incorporating gradients of population, trade, investment, and regulation, the stretch of shore between Houston, Texas and Mobile, Alabama presents one of the most vulnerable megaregions throughout the entirety of the United States. Annual land loss exposes urban cores and embedded industries to increased risk as the wetland fabric that formerly sheltered the region subsides. Amongst the silts of the Mississippi River Delta, contradictions between human and geologic time become visible; static methods to quantify, control, and value land fail in an 
environment that is constantly in flux. Links between urban environments and economic drivers have constrained the dynamic delta landscape for generations, now threatening to undermine the ecological fitness of the entire region. In order to defend settlement and economy across the Gulf Coast States, the integration of infrastructures must be reconsidered and agile forms of implementation should arise in place of stagnant control systems. In these complex territories, land is a resource for mitigating risk; multiscalar megaregional strategies, structural as well as nonstructural design projects, and flexible systems of implementation provide support systems that consider and assimilate environmental fluctuations in an efficient manner.

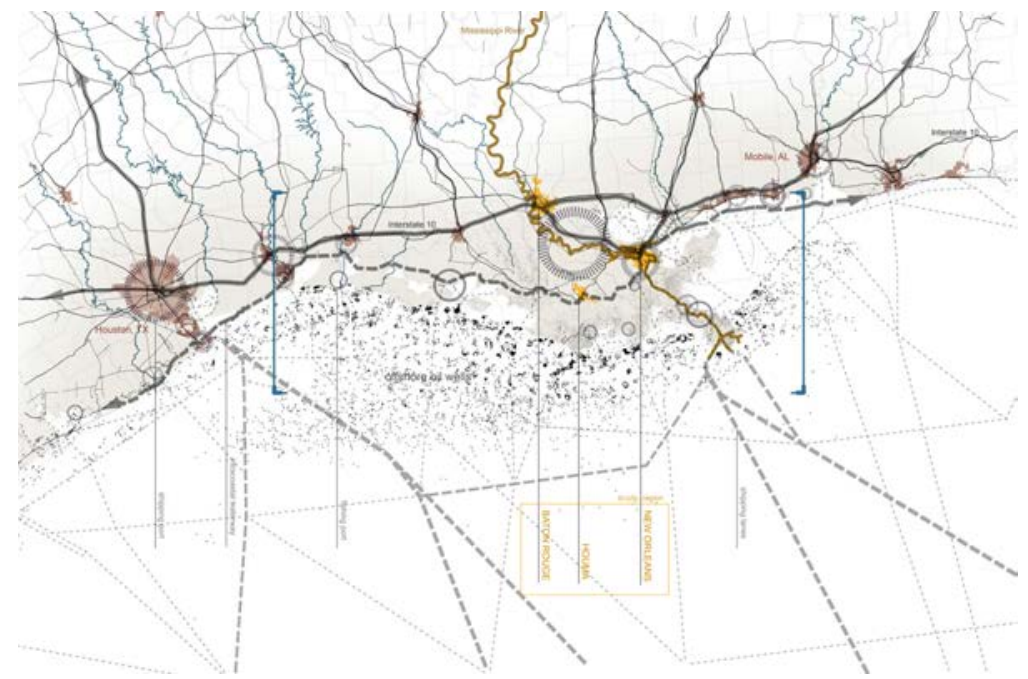

\section{Ecological Valuation : Risk Mitigation Land Benefits}

\section{Infrastructures of the Gulf Coast Megaregion}

Correlations between municipalities at neighbourhood, city, state, and regional scales evolve through and alongside environmental, economic, and industrial systems. The infrastructures that sustain these geographic expanses cross jurisdictions and boundaries, complicating the already interwoven fabrics of human settlement; however, the borders between urban, suburban, and rural environments are increasingly difficult to define, gradually eroding into density spectrums of continually populated landscapes. Along the federally funded and managed Interstate-10 [l-10] corridor, the southeast portion of the U.S. has emerged as an economically significant and industrially valuable territory. The geographic section between east Texas and south Alabama renders an increasingly intertwined urban ecology of shipping, petrochemical, and transportation infrastructures. South of this federal highway, local roads, ports, and railways are increasingly vulnerable; communities, industries, and their support structures are at risk of frequent or permanent inundation due to subsidence and coastal erosion. A 6 meter storm surge would flood $100 \%$ of the roads south of the I-10 passage. In order to defend infrastructure, economy, and settlement within the region, this project proposes that we must re-establish a rapidly disappearing landscape by constructing new land to alleviate increasing risk.

Coastal vegetation has been shown to significantly affect wave attenuation as measured by reductions in wave height per unit distance across a wetland.

Deteriorating wetland environments can impact the storm intensity that reaches inland environments. Across a geographic area, the physical functions provided by wetland environments may be similar; however, valuable services to populations vary according to density of those urban conditions and the value those communities ascribe to them. Through ecosystem services valuation methods, natural capital is ascribed to the conditions and processes of natural ecosystems and species that sustain and fulfill human life. These measurable benefits are especially considerable in terms of saltwater inundation and risk reduction. Capable of reducing the overall height of storm surge, physically slowing the forward momentum of the storm, and preventing hurricanes from pulling up more water, wetland environments are capable of providing direct and indirect value to gradients of urban conditions and the surroundings that perpetuate them. 


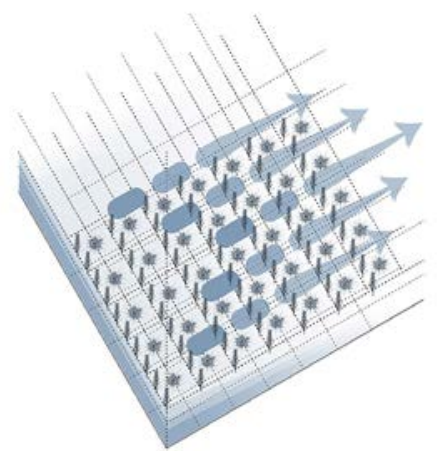

- - + Prevent Accumulation: deprive ability of storm to pull up more water

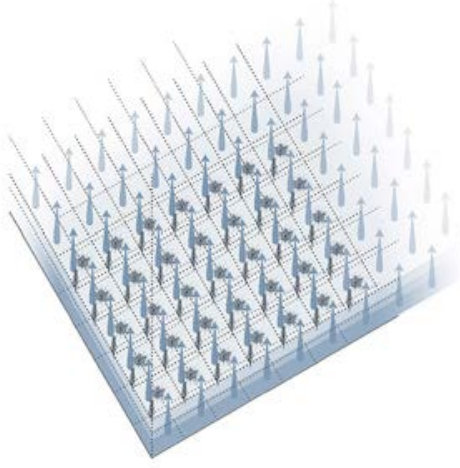

- - + Decrease Velocity: slow movement forward allowing for dissipation

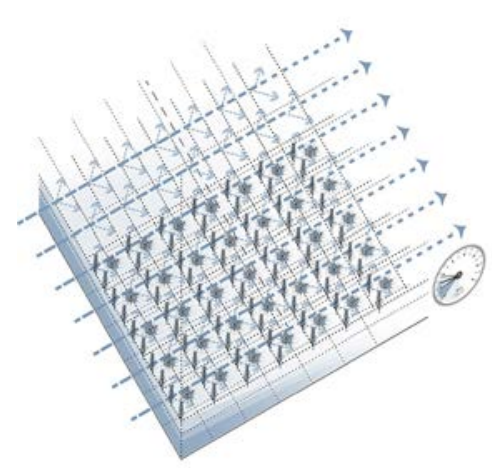

- - + Height Reduction : reduce volume with drag of vegetation

With the incorporation of structural and nonstructural protection systems, discrepancies in rates of deterioration, change, growth, and loss are continually present. In order to better understand the conditions as they change, continually updated datasets are required. Populations and the risks in which they are submerged continue to shift; however, regularly measuring those variables and designing their relationships, infrastructure design strategies can better understand how those varied forces overlay and interact. Population density surveys allow for an understanding of risk where sizeable communities influence reduction and response practices. Structural and nonstructural protection system diagrams illustrate design projects as they pass through stages of planning, construction, implementation, and evolution. The ability for wetland vegetation to influence risk can be partially determined by species and habitat structure; the ecosystem strength, durability, and change must be continually monitored in order to determine the value they presently provide to the surrounding areas as well as influencing future points of action. Through the Coastal Reference Monitoring System, wetland loss and restoration efforts are observed in order to understand the cumulative effectiveness of projects. This existing infrastructure assists in observation and recording of these changing conditions; however, the spatial distribution of these sites could be densified in order to better assess and quantify the transient landscape.

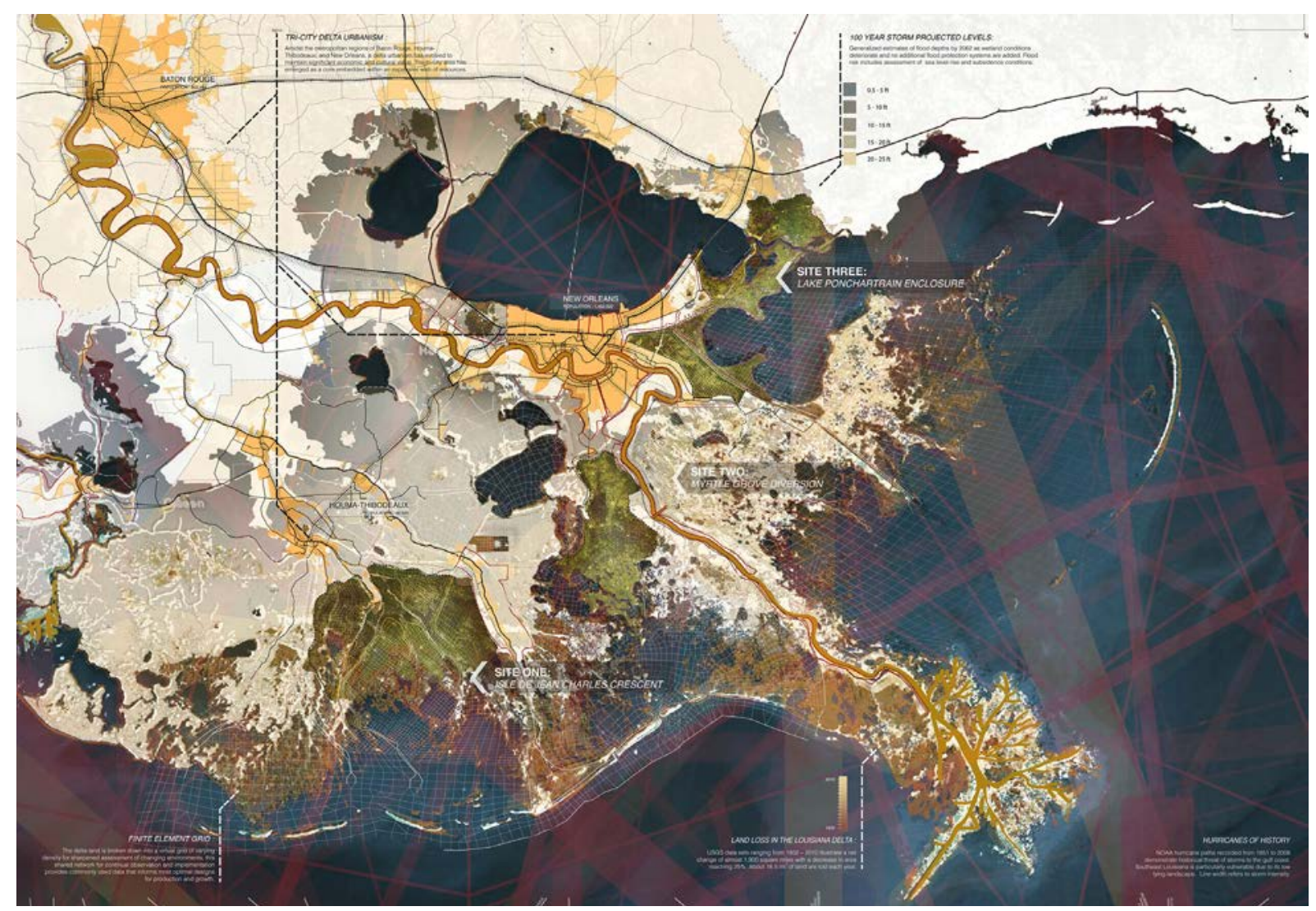

Risk Mitigation for the Urban Ecology; Depicting gradients of exposure to the Tri-City Delta urban core, storm surge probability, historic hurricane tracks, and land loss over the better part of the last century are overlayed to illustrate the project sites within the larger Southeast Louisiana context. 
Within the urban-economic expanse of the Gulf Coast Megaregion, the tri-city delta landscape between New Orleans, Baton Rouge, and Houma-Thibodeaux has evolved to maintain significant cultural and industrial value; the central wetland of Louisiana is embedded within this expansive web of resources. Land loss and storm surge datasets continue to assist in determining optimal locations for structural and nonstructural infrastructure implementation across southeast Louisiana. In order to found a resilient, layered mitigation strategy, Synthetic Mudscapes envisions three distinct land building practices; this set of strategies provides an arc of transforming systems to fortify aforementioned spectrums of inhabitation. Within the Isle de Jean Charles Crescent, dredge material and drill cutting debris are deposited nearby continually deepened transportation lanes and expanding oil fields; high diversity and concentrated seeding is dispersed to sustain land in material repositories. Expanding from an outlet in the Mississippi River Levee System, the Myrtle Grove Diversion releases sediment and shifted landfill medium transported from the river basin is deposited to accelerate natural land building processes. Combining treated sewage for wetland fertilization as well as wave attenuation arrays in nearby open waters, the Lake Ponchartrain Enclosure evolves as a constructed topography incorporating strategic infill of repurposed waste from nearby urban areas. Each of these strategies reintroduces fluctuation and adaptive management in order to form the foundations of protective, fertile fabrics.

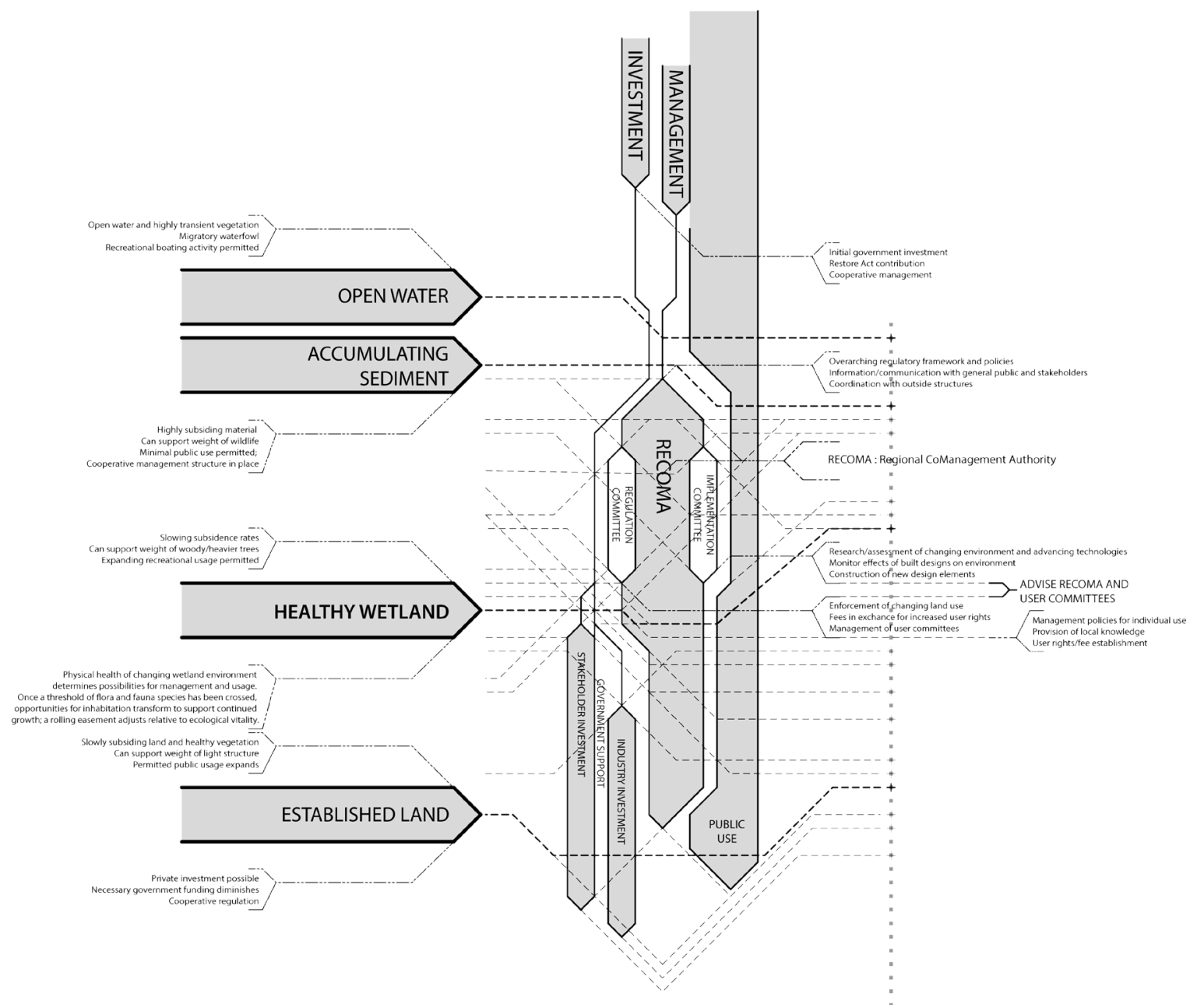

Ecosystem Service Dependent Landscape Use Strategy 


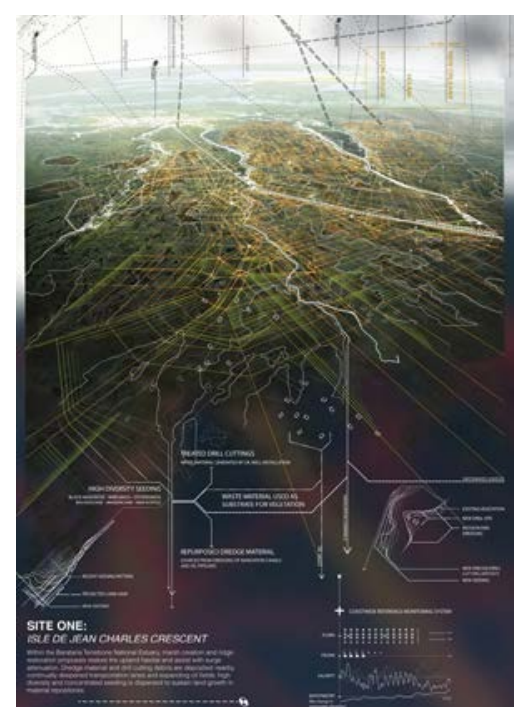

Land Building Site 1: Isle de Jean Charles Crescent; Within the Barataria Terrebonne National Estuary, marsh creation and ridge restoration proposals restore the upland habitat and assist with surge attenuation. Dredge material and drill cutting debris are deposited nearby continually deepened transportation lanes and expanding oil fields; high diversity and concentrated seeding is dispersed to sustain land growth in material repositories.

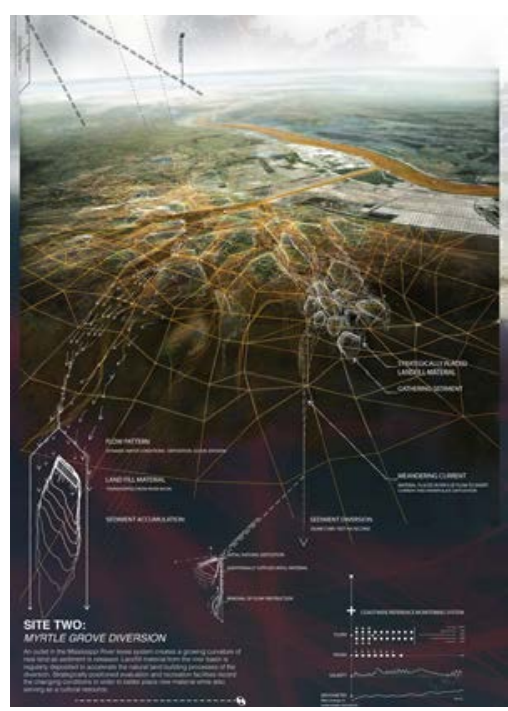

Land Building Site 2: Myrtle Grove Diversion; An outlet in the Mississippi River levee system creates a growing curvature of new land as sediment is released. Landfill material from the river basin is regularly deposited to accelerate natural land building processes as strategically placed evaluation and recreation facilities record the changing conditions in order to better place new material while also serving as a cultural resource.

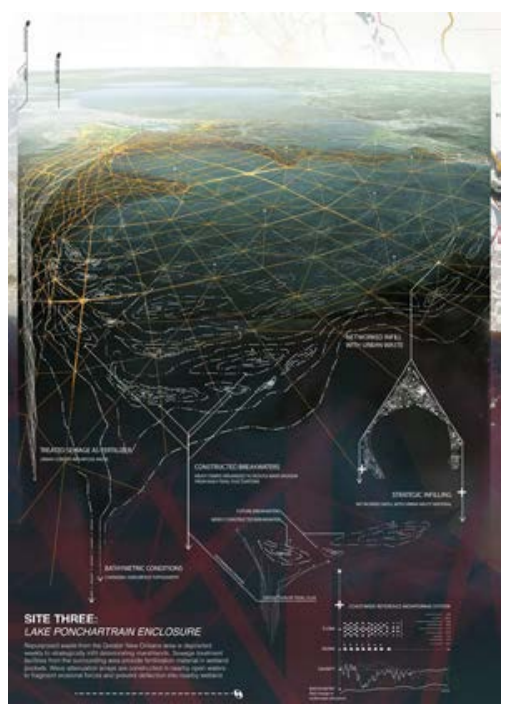

Land Building Site 3: Lake Ponchartrain Enclosure; Repurposed waste from the Greater New Orleans area is deposited weekly to strategically infill deteriorating marshlands. Sewage treatment facilities from the surrounding area provide fertilization material in wetland pockets while wave attenuation arrays are constructed in nearby open waters to fragment erosional forces and prevent deflection into fragile, nearby marshes.

The possibility for considering such methods of transformative infrastructures lies in the capacity to understand environments as they transform; through a latticed network of spatial nodes, a finite element grid establishes a framework for constant methods of measurement in a continually shifting environment. Changes and developments in urban ecosystems are registered and the system projects viability estimations and success rates of reconstructive design processes; the density of the mesh communicates situational variances in graphically illustrated detail. Data acquisitions and incorporations allow for interplays between distant information and related design decisions; deposition and integration strategies are based on continually monitored ecosystem vitality at the scale of inhabitation. Information acquired through satellite locations provides the capacity for virtual cartographies to become embedded with layers of relevant information; data collection becomes a tactic for understanding relationships between scales in time and the landscape. Considerate assimilation of these datasets promotes the integration of design and implementation practices across the varied delta landscape; a resilient, urban, coastal ecosystem arises from understandings of interwoven industrial, ecological, economic, and regulatory relationships.

While effectiveness of infrastructure projects is continually measured, an investment and management strategy is implemented to respond to the dynamism of the urban ecology. As the surface area of land changes, varied methods of financing and occupation are put in practice to fund transforming infrastructures. Initial investments of federal, state, and local government are followed by possibilities of shared occupation and rights to the land as a resource; multiple users contribute relative to function for maintenance and growth. As variations in wetland environments shift between open water and established land, the quality and physical condition of the ecosystem determines its usage; public and industrial utilization is accepted at stronger, healthier stages of ecosystem development. However, as territories remain within a delicate stage of accumulating sediment, the overarching regulatory framework and policies reflect the significance of future risk reducing interests via stakeholders and the general public. Changing use and increased user rights accompanies management policies with newly established or re-established land. Questions of utilization and occupation are addressed through an adjustable support structure.

From the cores of urban areas to the surroundings that sustain them, continually changing environments require infrastructures that can consider and assimilate environmental fluctuations efficiently. Reflexive practices that integrate 
transdisciplinary proposals with a monitored understanding of conditions as they change, flexible investment and management practice, and adaptive implementation strategies can be deployed across scales in a considerate and particular manner. Multiscalar processes require agile infrastructures to respond to increased risk and a variety of design practices can be introduced within those landscapes. Additionally, including land growth in contrast to the construction of a floodwall, variations in time frames for actualization must be considered throughout project development and deployment. Structural and nonstructural projects can be assimilated in dialogue through data acquisition and continual communication between design and planning bodies. Relationships between populations and the environments they inhabit are in constant flux; being so, it is necessary that the tactics we use to invest in, construct, and manage these processes reflect this continual change. Rising seas, subsidence, and coastal erosion are reorganizations that are constantly evolving and often relatively unpredictable; linear methods of working are incapable of functioning in these complex territories and are ill equipped to respond to these recurring transformations. Megaregional infrastructural systems traverse borders and boundaries, industries, economies, cultures and populated jurisdictions; understanding how infrastructures affect and are affected by the geographies they support is necessary to advance our interwoven, urban ecologies. In order to satisfy the requirements of the diverse demographically and industrially inhabited megaregion, the complex natural environments in which they are embedded must be continually addressed. 